\title{
Doenças Crônicas não Transmissíveis em Usuários de Unidades de Saúde da Família - Prevalência, Perfil Demográfico, Utilização de Serviços de Saúde e Necessidades Clínicas
} Chronic Noncommunicable Diseases Among Family Health Unit Users -
Prevalence, Demographics, Use of Health Services and Clinical Needs

\author{
TATIANA DE OLIVEIRA SATO ${ }^{1}$ \\ NATHALYATAMARA COSTA FERMIANO2 \\ MARIANA VIEIRABATISTÃO ${ }^{3}$ \\ ANA SILVIA MOCCELLIN ${ }^{4}$ \\ PATRICIADRIUSSO ${ }^{1}$ \\ SÍLVIA HELENAZEM MASCARENHAS ${ }^{5}$
}

\section{RESUMO}

Objetivo: Avaliar a prevalência de doenças crônicas não transmissíveis (DCNT) entre usuários adultos e idosos de Unidades de Saúde da Família do município de São Carlos/SP e identificar o perfil demográfico, utilização de serviços de saúde e as necessidades clínicas destes usuários. Materiais e métodos: O estudo foi desenvolvido em 16 USF do município de São Carlos/SP, incluindo 2407 famílias e 8844 pessoas. Dados sobre a prevalência de DCNT foram analisados para a população adulta (maior de 18 anos; $n=5690$ ). Variáveis demográficas foram analisadas para os grupos com DCNT e controle. Os dados foram analisados descritivamente e por meio do teste Qui-quadrado no programa SPSS com nível de significância de $5 \%$. Resultados: Os resultados mostraram que a prevalência de DCNT foi de aproximadamente $35 \%$ (IC95\%=33,4-35,9\%), sendo maior em idosos, mulheres, indivíduos com menor grau de escolaridade, viúvos, aposentados e afastados do trabalho. Não houve associação entre as DCNT e o tabagismo. O serviço de saúde mais utilizado foi a USF em ambos os grupos. Indivíduos com DCNT utilizaram com maior frequência a Unidade de Pronto Atendimento, Unidade Básica de Saúde e Centro de Especialidades Médicas. Conclusões: Os resultados demonstraram que a prevalência de DCNT é alta entre os adultos e idosos avaliados neste estudo, o Sistema Único de Saúde é a referência para tratamento/acompanhamento desses indivíduos. Ações de planejamento para prevenção e controle das DCNT podem ser delineadas no município a partir deste perfil.

\section{DESCRITORES}

Sistema Único de Saúde. Saúde da Família. Epidemiologia. Prevenção. Hipertensão Arterial Sistêmica. Diabetes.

\begin{abstract}
Objective: To evaluate the prevalence of chronic noncommunicable diseases (NCD) among adults and elderly users of the Family Health Units (FHU) in São Carlos/SP and identify the demographics, use of health services and clinical needs of these users. Material and Methods: The study was conducted in $16 \mathrm{FHU}$ in São Carlos/SP, including 2,407 families and 8,844 individuals. The NCD prevalence data were analyzed for the adult population (above 18 years; $n=5,690$ ). Demographic variables were analyzed for the groups with NCD and control. The data were analyzed descriptively and by the Chi-square test of association in SPSS program, with a $5 \%$ significance level. Results: The results showed that the prevalence of NCD was approximately $35 \%(95 \% \mathrm{Cl}=33.4$ to $35.9 \%$ ), being higher in the elderly, women, individuals with lower education, widowed and retired/off work. There was no association between smoking and NCD. The most used health service was the FHU in both groups. Individuals with NCD used most frequently the Emergency Unit, Basic Health Unit and Center for Medical Specialties. Conclusions: The results showed that the prevalence of NCD is high among the adults and elderly evaluated in this study, and that the Brazilian Public Health System is the basis for treatment/ follow-up of these individuals. Based on our data, planning actions aimed at prevention and control of NCD can be performed in the context of Primary Health Care in this municipality.
\end{abstract}

DESCRIPTORS

Unified Health System. Family Health. Epidemiology. Prevention. Systemic Arterial Hypertension. Diabetes.

\footnotetext{
Professor. Departamento de Fisioterapia, Universidade Federal de São Carlos. São Carlos/São Paulo (SP), Brasil Graduando. Departamento de Fisioterapia, Universidade Federal de São Carlos. São Carlos/São Paulo (SP), Brasil Doutorando. Departamento de Fisioterapia, Universidade Federal de São Carlos. São Carlos/São Paulo (SP), Brasil Professor. Departamento de Fisioterapia da Universidade Federal de Sergipe. São Cristóvão/Sergipe (SE), Brasil.
}

Professor. Departamento de Enfermagem, Universidade Federal de São Carlos. São Carlos/São Paulo (SP), Brasil 
A s doenças crônicas não transmissíveis (DCNT) são um problema de saúde pública tanto nos países desenvolvidos quanto nos países em desenvolvimento. A Organização Mundial da Saúde (OMS) define como DCNT as doenças cardiovasculares, respiratórias crônicas, câncer e diabetes mellitus ${ }^{1}$. Estas doenças se caracterizam por múltipla etiologia, curso prolongado, origem não infecciosa e associação com deficiências e incapacidades funcionais. Os fatores de risco para ocorrência das DCNT podem ser classificados em não modificáveis (sexo, idade, herança genética) e comportamentais (tabagismo, alimentação não saudável, sedentarismo, consumo de álcool e obesidade), sendo potencializados pelos aspectos socioeconômicos, culturais e ambientais ${ }^{1,2}$.

$\mathrm{Na}$ atualidade as DCNT representam um grande desafio aos sistemas de saúde. Dados dos 193 países membros da OMS indicam que mais de 36 milhões de pessoas morreram de DCNT em 2008, sendo $48 \%$ das mortes causadas por doenças cardiovasculares, 21\% câncer, $12 \%$ doenças respiratórias crônicas e 3\% diabetes. Além disso, 25\% destas mortes ocorreram em indivíduos com idade inferior a 60 anos e poderiam ter sido evitadas ${ }^{3}$.

Os efeitos dessas doenças podem ser notados na esfera econômica, uma vez que os custos com o tratamento são altos, causam incapacidade para o trabalho, absenteísmo e aposentadoria por invalidez ${ }^{4}$. Essas doenças comprometem a renda familiar e a economia, uma vez que aumentam as taxas de internação e consumo de medicamentos, onerando os cofres públicos ${ }^{5}$.

As DCNT demandam ações e procedimentos dos serviços de saúde, principalmente na atenção básica. Estes serviços possibilitam compromisso e responsabilização com as condições de vida e de saúde da comunidade, uma vez que realizam acompanhamento longitudinal baseado nos princípios da integralidade, qualidade e equidade, estabelecendo maior vínculo com a população ${ }^{4}$.

Mediante essas questões, a atenção básica vem se estruturando em busca da capacitação das equipes para conhecer a distribuição e a tendência das doenças crônicas e seus fatores de risco e apoiar as políticas públicas de promoção da saúde ${ }^{6}$. Assim, torna-se importante realizar diagnósticos que possam subsidiar as ações locais de enfrentamento dessa epidemia. O Ministério da Saúde elaborou um Plano de Ações Estratégicas para o Enfrentamento das Doenças Crônicas não Transmissíveis ${ }^{78}$ que visa combater as DCNT em dez anos (2011-2022), atuando nos quatro principais grupos de doenças (circulatórias, câncer, doenças respiratórias crônicas e diabetes) e seus fatores de risco (tabagismo, consumo nocivo de álcool, inatividade física, alimentação inadequada e obesidade). O plano define diretrizes e ações em vigilância, informação, avaliação e monitoramento, promoção da saúde e cuidado integral. Para que este plano tenha êxito diversas ações devem ser implementadas. Desta forma, surge como necessidade dos serviços realizarem inquéritos de saúde para conhecer a magnitude, abrangência e necessidades geradas pelas DCNT.

Diante disso, este estudo teve por objetivo avaliar a prevalência de doenças crônicas não transmissíveis entre usuários adultos e idosos adscritos às Unidades de Saúde da Família (USF) do município de São Carlos/SP e identificar o perfil demográfico, utilização de serviços de saúde e as necessidades clínicas destes usuários.

\section{MATERIAISE MÉTODOS}

Foi realizado um estudo observacional transversal a partir dos dados provenientes da pesquisa realizada no contexto do Programa de Educação pelo Trabalho para Saúde (PET-Saúde), realizado nas 16 Unidades de Saúde da Família do município de São Carlos/SP no período de 2009 a 2010. As 16 unidades representavam a totalidade das USF do município no período. Foram realizadas 2407 entrevistas domiciliares, totalizando 8844 indivíduos avaliados, sendo $5690 \mathrm{com}$ idade superior a 18 anos.

Foram definidos como participantes deste estudo os usuários adultos (idade $>18$ anos) cujas famílias estavam cadastradas no Sistema de Informação da Atenção Básica (SIAB). Foram excluídas as famílias que após três tentativas de contato, em dias e horários diferentes, não foram encontradas no domicílio; famílias que se recusaram a participar e famílias nas quais o respondente apresentava dificuldade de compreensão do questionário. 
Foi utilizado o QPET-UFSCar como instrumento de coleta de dados ${ }^{8}$. Este questionário foi desenvolvido pelos participantes do PET-Saúde, sendo composto por 55 questões que abordam aspectos pessoais, demográficos e necessidades clínicas.

Os dados do QPET-UFSCar foram coletados por estudantes da área da saúde, com apoio dos preceptores e tutores. A partir da listagem do número SIAB, o endereço das famílias sorteadas era localizado e os alunos realizavam a coleta de dados em domicílio. As entrevistas foram realizadas com a pessoa responsável pelo domicílio com idade superior a 18 anos. Cada entrevista durava em torno de 30 minutos. Os estudantes eram identificados com jaleco e crachá e inicialmente informavam ao usuário os objetivos da pesquisa e a não obrigatoriedade de participação. O usuário era informado explicitamente que a não participação não implicaria em qualquer ônus ou constrangimento perante a equipe de saúde. O estudo foi aprovado pelo Comitê de Ética e Pesquisa com Seres Humanos da UFSCar (Parecer n⿳0 247/2009-CAAE 1638.0.000.135-09).

Os formulários foram digitados no programa EPI INFO. Após a digitação procedeu-se uma análise da consistência dos resultados, sendo necessária verificação dos formulários digitados para checagem e correção de dados. Os dados foram analisados de forma descritiva, por meio do cálculo de valores médios, desvios padrão, frequências e intervalos de confiança, visando caracterizar a amostra.

Os usuários que possuíam DCNT (doenças cardiovasculares, respiratórias, musculoesqueléticas e diabetes mellitus) foram identificados e dois grupos foram compostos: grupo controle (sem DCNT) e caso (pelo menos uma DCNT). Realizou-se comparação entre os grupos para as seguintes variáveis: gênero, grupo etário (adulto ou idoso), escolaridade, estado civil, ocupação, condição de saúde, utilização de serviços de saúde e necessidades clínicas. A comparação entre grupos foi realizada por meio do teste de associação Qui-quadrado $\left(\div^{2}\right)$ com nível de significância de 5\% (á=0,05) no programa SPSS (versão 17.0).

\section{RESULTADOS}

Foram analisados os dados referentes a 5690 usuários, sendo que a prevalência de DCNT foi de 34,6\% (IC95\%=33,4-35,9\%) na amostra. A prevalência foi maior nos idosos (>60 anos) em relação aos adultos (18 a 59 anos). Além disso, a prevalência de DCNT foi maior no grupo feminino em relação ao masculino. Nota-se também maior prevalência nos grupos com menor escolaridade e nos viúvos. As associações entre a prevalência de DCNT e as variáveis demográficas foram significantes (Tabela 1).

A Tabela 2 mostra as ocupações relatadas pelos participantes dos grupos controle, caso e na amostra total. Pode-se notar que dentre os indivíduos do grupo caso é maior a proporção de aposentados/afastados; já dentre os indivíduos do grupo controle é maior a proporção de estudantes $(\div 2=644,21 ; p<0,001)$.

Na Figura 1 estão apresentadas a frequência relativa e os intervalos de confiança para as principais condições de saúde relatadas no grupo DCNT. Nota-se que os distúrbios cardiovasculares foram os problemas mais frequentes, seguidos dos musculoesqueléticos, diabetes e distúrbios respiratórios.

A Tabela 3 mostra os fatores de risco encontrados nos participantes dos grupos caso, controle e para amostra total. Não houve associação entre o tabagismo e o grupo DCNT $(p=0,69)$. O grupo controle apresenta maior proporção de pessoas que fazem uso nocivo do álcool $(p=0,01)$. Todas as necessidades clínicas investigadas foram mais frequentes no grupo caso $(p<0,05)$ (Tabela 3 ).

O grupo caso utiliza a USF com maior regularidade do que o grupo controle. O serviço de saúde mais utilizado por ambos os grupos é a USF, seguido da Unidade de Pronto Atendimento (UPA) e Unidade Básica de Saúde (UBS). O grupo caso utiliza mais a UPA, UBS e Centro de Especialidade Médicas do que os sujeitos do grupo controle. Nota-se ainda que a proporção de indivíduos que possuem convênio médico particular é maior no grupo controle $(p<0,001)$ (Tabela 4).

\section{DISCUSSÃO}

Os resultados do presente estudo indicam prevalência de indivíduos adultos e idosos com DCNT de aproximadamente $35 \%$, sendo maior em idosos, mulheres, indivíduos com menor grau de escolaridade, 


\begin{tabular}{lcccc}
\multicolumn{5}{l}{$\begin{array}{l}\text { Tabela 1. Dados demográficos para o grupo controle, caso e para amostra total. Dados } \\
\text { estão apresentados em frequência absoluta e relativa In(\%)I.São Carlos/SP, } 2009\end{array}$} \\
$\begin{array}{l}\text { Características } \\
\begin{array}{l}\text { Gênero } \\
\text { Masculino }\end{array}\end{array}$ & $\begin{array}{c}\text { Controle } \\
\text { Caso }\end{array}$ & \multicolumn{1}{c}{ Total } & $\boldsymbol{p}^{*}$ \\
$\begin{array}{l}\text { Feminino } \\
\text { Grupo Etário }\end{array}$ & $1913(68,6)$ & $877(31,4)$ & $2790(49,0)$ & $<0,001$ \\
Adulto & $1811(62,4)$ & $1089(37,6)$ & $2900(51,0)$ & \\
Idoso & & & & $<0,001$ \\
Escolaridade & $3632(72,2)$ & $1397(27,8)$ & $5029(85,8)$ & \\
Nenhuma & $200(24,0)$ & $633(76,0)$ & $833(14,2)$ & \\
Ensino Fundamental & $140(36,1)$ & $248(63,9)$ & $388(7,7)$ & \\
Ensino Médio & $1022(51,3)$ & $969(48,7)$ & $1991(39,3)$ & \\
Ensino Superior & $1735(79,8)$ & $439(20,2)$ & $2174(40,9)$ & \\
Técnico & $85(73,3)$ & $31(26,7)$ & $116(2,3)$ & \\
Estado Civil & $329(82,2)$ & $71(17,8)$ & $400(7,9)$ & \\
Viúvo & & & & $<0,001$ \\
Separado/Divorciado & $83(25,9)$ & $238(74,1)$ & $321(5,5)$ & \\
Casado/União Estável & $216(56,8)$ & $164(43,2)$ & $380(6,5)$ & \\
Solteiro & $2418(64,0)$ & $1363(36,0)$ & $3781(64,8)$ & \\
\hline
\end{tabular}

\begin{tabular}{|c|c|c|c|}
\hline Ocupação & $\begin{array}{c}\text { Controle } \\
n=3819(65,4 \%)\end{array}$ & $\begin{array}{c}\text { Caso } \\
n=2013(34,6 \%)\end{array}$ & $\begin{array}{c}\text { Total } \\
n=5831(100,0 \%)\end{array}$ \\
\hline $\begin{array}{l}\text { Aposentado/Afastado } \\
\text { Catador } \\
\text { Do Lar } \\
\text { Cuidador/Babá } \\
\text { Empregada Doméstica } \\
\text { Aaricultura } \\
\text { Desempreqado } \\
\text { Construcão Civil } \\
\text { Motorista } \\
\text { Autônomo } \\
\text { Não Informado } \\
\text { Serviços } \\
\text { Comércio } \\
\text { Funcionário Público } \\
\text { Escritório } \\
\text { Professor } \\
\text { Nível Superior } \\
\text { Indústria } \\
\text { Estudante }\end{array}$ & $\begin{array}{l}201(29,2) \\
17(53,1) \\
493(55,4) \\
22(61,1) \\
233(61,3) \\
84(65,1) \\
246(68,1) \\
180(69,4) \\
95(69,8) \\
219(69,9) \\
160(73,7) \\
824(75,5) \\
275(77,0) \\
26(78,7) \\
72(80,0) \\
40(80,0) \\
29(80,5) \\
491(81,1) \\
97(88,9)\end{array}$ & $\begin{array}{l}487(70,7) \\
15(46,8) \\
396(44,5) \\
14(38,8) \\
147(38,6) \\
45(34,8) \\
115(31,8) \\
79(30,5) \\
41(30,1) \\
94(30,0) \\
57(26,2) \\
266(24,4) \\
82(22,9) \\
7(21,2) \\
18(20,0) \\
10(20,0) \\
7(19,4) \\
114(18,8) \\
12(11,0)\end{array}$ & $\begin{array}{l}688(11,7) \\
32(0,5) \\
889(15,2) \\
36(0,6) \\
380(6,5) \\
129(2,2) \\
361(6,1) \\
259(4,4) \\
136(2,3) \\
313(5,3) \\
217(3,7) \\
1090(18,6) \\
357(6,1) \\
33(0,5) \\
90(1,5) \\
50(0,8) \\
36(0,6) \\
605(10,3) \\
109(1,8)\end{array}$ \\
\hline
\end{tabular}




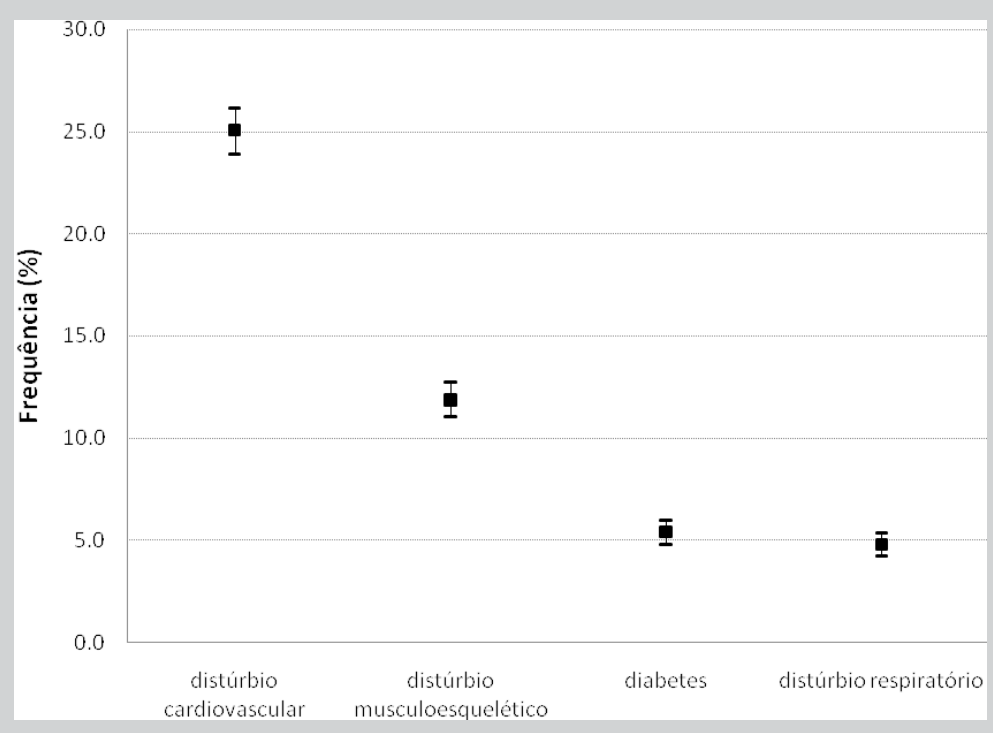

Figura 1. Prevalência e Intervalo de confiança (95\%) das condições de saúde comuns no grupo com doenças crônicas não transmissíveis. São Carlos/SP, 2009

\begin{tabular}{|c|c|c|c|c|}
\hline Característica & Controle & Caso & Total & $p^{*}$ \\
\hline \multicolumn{5}{|l|}{ Fatores De Risco } \\
\hline Tabagismo & $839(22,3)$ & $437(21,8)$ & $1276(22,1)$ & 0,69 \\
\hline Consumo Nocivo De Álcool & $643(17,3)$ & $284(14,4)$ & $927(16,3)$ & 0,01 \\
\hline \multicolumn{5}{|l|}{ Necessidades Clínicas } \\
\hline Cessar Tabaqismo & $398(10.4)$ & $252(12,4)$ & $650(11,1)$ & 0.019 \\
\hline Realizar Intervenção Clínica & $410(10,7)$ & $460(22,7)$ & $870(14,8)$ & $<0,001$ \\
\hline Usar Medicação Correta & $26(0,7)$ & $75(3,7)$ & $101(1,7)$ & $<0,001$ \\
\hline Cessar Consumo De Álcool & $135(3,5)$ & $98(4,8)$ & $233(4,0)$ & 0,025 \\
\hline Praticar Atividade Física & $414(10,8)$ & $285(14,0)$ & $699(11,9)$ & 0,001 \\
\hline Realizar Reeducacão Alimentar & $162(4,2)$ & $132(6,5)$ & $294(5,0)$ & $<0,001$ \\
\hline Melhorar Adesão Ao Tratamento & $154(4,0)$ & $128(6,3)$ & $282(4,8)$ & $<0,001$ \\
\hline Referência Para Nível Especializado & $90(2,3)$ & $137(6,7)$ & $227(3,9)$ & $<0,001$ \\
\hline
\end{tabular}




\begin{tabular}{|c|c|c|c|c|}
\hline Utilização Dos Serviços (N [\%]) & Controle & Caso & Total & $p^{*}$ \\
\hline Unidade De Saúde Da Família & & & & $<0,001$ \\
\hline Reqularmente & $1158(30,4)$ & $1078(53,2)$ & $2236(38,3)$ & \\
\hline Esporadicamente & $1619(42,5)$ & $659(32,5)$ & $2278(39,0)$ & \\
\hline Não Usa & $1036(27,2)$ & $289(14,3)$ & $1325(22,7)$ & \\
\hline Serviços Mais Utilizados & & & & 0,004 \\
\hline Unidade De Saúde Da Família & $1053(55,3)$ & $155(51,0)$ & $1208(54,7)$ & \\
\hline Unidade De Pronto Atendimento & $231(12,1)$ & $42(13,8)$ & $273(12,4)$ & \\
\hline Unidade Básica De Saúde & $210(11.0)$ & $44(14,5)$ & $254(11.5)$ & \\
\hline Consultório Privado & $209(11,0)$ & $31(10,2)$ & $240(10,9)$ & \\
\hline Hospital & $126(6,6)$ & $21(6,9)$ & $147(6,7)$ & \\
\hline Outro & $58(3,0)$ & $2(0,7)$ & $60(2,7)$ & \\
\hline Centro De Especialidades Médicas & $17(0,9)$ & $9(3,0)$ & $26(1,2)$ & \\
\hline \multirow[t]{2}{*}{ Possui Convênio Médico Particular } & & & & $<0,001$ \\
\hline & $786(20,7)$ & $324(16,0)$ & $1110(19,1)$ & \\
\hline
\end{tabular}

${ }^{*}$ Qui-quadrado $\left(x^{2}\right)$.

viúvos, aposentados/afastados. Assim, pode-se traçar um perfil demográfico dos indivíduos acometidos por estas doenças, que é compatível com dados disponíveis na literatura ${ }^{9-11}$.

A alta prevalência de DCNT em idosos se deve ao aumento da expectativa de vida uma vez que a redução da mortalidade por outras doenças fez com que os indivíduos ficassem mais expostos às $\mathrm{DCNT}^{12}$. Apesar de o processo de envelhecimento não estar relacionado necessariamente a doenças e incapacidades, as DCNT são frequentemente encontradas entre os idosos ${ }^{13}$.

A maior prevalência de DCNT no grupo feminino também foi encontrada em outros estudos nacionais ${ }^{9,11,14,15}$, sendo que este achado tem sido atribuído ao maior conhecimento dos sinais e sintomas, maior solicitação dos serviços de saúde e maior relato dos problemas de saúde por parte das mulheres em comparação aos homens ${ }^{11}$. Homens relatam menos os problemas que apresentam e solicitam com menos frequência os serviços de saúde. Associado a isto, sua percepção sobre a saúde é pior ao relatar a presença de DCNT, isso pode ser atribuído à demora em procurar o auxílio, buscando os serviços de saúde em estágios mais avançados da doença ${ }^{9}$.

A escolaridade é outro fator que interferiu na prevalência das DCNT, sendo que quanto menor o grau de instrução maior a prevalência de DCNT. Estes achados podem estar associados ao fato de que o menor nível de conhecimento das doenças e de seus fatores de risco prejudica a prevenção das DCNT. Pessoas com maior nível de escolaridade tendem a realizar mais atividade física, ingerir alimentos mais saudáveis e fazem menos uso do tabaco, que são os principais fatores de proteção para as DCNT. Além disso, melhores condições socioeconômicas também foram associadas ao estilo de vida mais saudável ${ }^{17}$.

Em relação à ocupação, dentre os indivíduos com DCNT é maior a proporção de aposentados e afastados do trabalho, já dentre os indivíduos do grupo controle é maior a proporção de estudantes. Estes achados possivelmente estão associados à idade dos indivíduos, assim como a maior prevalência encontrada nos indivíduos viúvos. Além disso, pode-se supor que as DCNT estejam também associadas ao afastamento do trabalho e aposentadoria por invalidez, onerando ainda mais o sistema de saúde e a previdência social ${ }^{4}$.

Não houve associação das DCNT com o tabagismo, apesar de o tabaco ser considerado um dos fatores de risco para desenvolvimento dessas doenças ${ }^{2}$. A proporção de tabagistas foi similar entre os grupos, em torno de $22 \%$. Em um estudo em 18 capitais brasileiras a prevalência foi de $20,8 \%{ }^{18}$. Os resultados de pesquisas recentes sugerem que as campanhas contra o tabagismo têm sido efetivas, sendo que o consumo de cigarro diminuiu entre os anos 1989 e 2003, de 34,8\% para $22,4 \%{ }^{19}$. 
A prevalência de consumo nocivo de álcool deste estudo é similar a outro estudo ${ }^{20}$, que analisou as informações de entrevistas telefônicas com a população adulta nas 26 capitais de estados brasileiros e no Distrito Federal e encontrou uma prevalência de consumo de risco de $17,6 \%$. No presente estudo, o grupo controle apresentou maior proporção de pessoas que usam o álcool de forma nociva em relação ao grupo de pessoas com DCNT. O consumo abusivo está associado à maior morbidade e mortalidade. Estima-se que entre os óbitos causados por álcool mais de $50 \%$ sejam devido às DCNT, incluindo diversos tipos de câncer, doença cardiovascular e cirrose hepática ${ }^{3}$. No entanto, devemos levar em consideração a confiabilidade do auto-relato, pois que a informação pode ser distorcida pelo entrevistado, pois ele deseja responder algo que seja socialmente desejável. A confiabilidade teste-reteste das informações auto relatadas de consumo de álcool é moderada ${ }^{21}$.

Segundo as informações da PNAD, 29,9\% da população brasileira reportou ter pelo menos, uma doença crônica em $2003^{22}$. As prevalências relatadas na literatura científica são semelhantes às encontradas neste estudo para distúrbios musculoesqueléticos (14\%), diabetes (9\%) e problemas respiratórios $(5 \%)^{23}$.

As necessidades clínicas investigadas como: realizar intervenção clínica, fazer uso de medicação, realizar reeducação alimentar, obter encaminhamento para nível especializado, foram maiores no grupo com DCNT. Este resultado era esperado, pois é da característica da doença a necessidade de acompanhamento contínuo. Segundo a literatura o envelhecimento e a presença de doenças crônicas ocasiona aumento do número de consultas, maior consumo de medicamentos, exames complementares e hospitalizações ${ }^{24,25}$.

Nesse mesmo sentido, em nosso estudo, notase um padrão de maior procura por atendimentos nos indivíduos com DCNT com maior frequência na Unidade de Saúde da Família. Da mesma forma houve maior busca por atendimento na Unidade de Pronto Atendimento, Unidades Básicas de Saúde, Centro de Especialidade Médicas e Hospital no grupo com DCNT. Em contraposição, a proporção de indivíduos que possuem convênio médico particular ou realizam consultas particulares é maior no grupo controle. Esse padrão indica uma sobrecarga ao sistema público de saúde, pois os custos no atendimento desses pacientes são muito altos.

Portanto, investir em ações para prevenir as DCNT é altamente necessário, pois preconizar o controle dos principais fatores de risco pode garantir que as gerações futuras não sofram o risco de morrer precocemente por causa dessas doenças, além de reduzir os altos custos gerados. O conhecimento sobre a abrangência das DCNT no contexto brasileiro e principalmente em nível municipal faz parte das ações necessárias para o combate mais efetivo destas doenças, evitando maior sobrecarga ao SUS.

\section{Limitações do estudo}

Por se tratar de um levantamento abrangente não foi possível aprofundar a avaliação dos fatores de risco. Além disso, algumas questões podem ter sofrido influência por se tratar de condutas socialmente não aceitáveis. Outra limitação se refere à forma de aplicação do questionário à pessoa responsável pelo domicílio que pode não ter conhecimento preciso sobre as informações de cada um dos membros da família.

\section{AGRADECIMENTOS}

Ao Conselho Nacional de Desenvolvimento Científico e Tecnológico pelo apoio financeiro concedido; à Secretaria de Gestão do Trabalho e Educação na Saúde (SGTES) do Ministério da Saúde pelo apoio oferecido por meio do Programa de Educação pelo Trabalho para Saúde (PET-Saúde), à Secretaria de Saúde da Prefeitura Municipal de São Carlos e aos profissionais e usuários das Unidades de Saúde da Família.

Fonte de financiamento: Programa de Educação pelo Trabalho para Saúde (PET-Saúde), Secretaria de Gestão do Trabalho e da Educação na Saúde (SGTES), Ministério da Saúde (MS), Brasil (Proc. N. 25000.666709/2009-40); Conselho Nacional de Desenvolvimento Científico e Tecnológico (Proc. N. 472552/2012-4). 


\section{REFERÊNCIAS}

1. World Health Organization. Preventing chronic diseases: a vital investment. Geneva; 2005.

2. Schmidt MI, Duncan BB, Azevedo e Silva G, Menezes AM, Monteiro CA, Barreto SM, et al. Chronic noncommunicable diseases in Brazil: burden and current challenges. Lancet 2011; 377(9781):1949-61.

3. World Health Organization. Noncommunicable diseases country profiles 2011. Geneva; 2011.

4. Brasil. Ministério da Saúde. Secretaria de Vigilância em Saúde. Política Nacional de Promoção da Saúde. Brasília; 2010.

5. Brasil. Ministério da Saúde. A vigilância, o controle e a prevenção das doenças crônicas não transmissíveis: DCNT no contexto do Sistema Único de Saúde Brasileiro. Brasília; 2005

6. Paim J, Travassos C, Almeida C, Bahia L, Macinko J. The Brazilian health system: history, advances, and challenges. Lancet 2011;377(9779):1778-97.

7. Brasil. Ministério da Saúde. Plano de ações estratégicas para o enfrentamento das doenças crônicas não transmissíveis (DCNT) no Brasil 2011-2022. Brasília: Ministério da Saúde, 2011.

8. Zem-Mascarenhas SH, Fabbro MRC, Floriano PJ, Joaquim RVT, França Y, Sato TO. Relatório técnico final das atividades do PET-Saúde/Saúde da Família UFSCar/SMS São Carlos. 2010

9. Barreto SM, Figueiredo RC. Doença crônica, autoavaliação de saúde e comportamento de risco: diferença de gênero. Rev Saúde Pública 2009; 43(Supl 2):38-47.

10. Cavalcanti CL, Gonçalves MCR, Asciutti LSR, Cavalcanti AL. Prevalência de doenças crônicas e estado nutricional em um grupo de idosos brasileiros. Rev Salud Pública 2009; 11(6):865-77.

11. Barros MBA, César CLG, Carandina L, Torre GD. Desigualdades sociais na prevalência de doenças crônicas no Brasil, PNAD-2003. Ciência Saúde Coletiva 2006; 11(4):911-26.

12. Achutti A, Azambuja MIR. Doenças crônicas nãotransmissíveis no Brasil: repercussões do modelo de atenção à saúde sobre a seguridade social. Ciência Saúde Coletiva 2004; 9(4):833-40.

13. Veras RP, Caldas CP, Coelho FD, Sanchez MA. Promovendo a saúde e prevenindo a dependência: identificando indicadores de fragilidade em idosos independentes. Rev Bras Geriat Geront.2007;10(3):35570.

14. Almeida MF, Barata RB, Montero CV, Silva ZP. Prevalência das doenças crônicas auto-referidas e utilização dos serviços de saúde PNAD/1998, Brasil. São Paulo 1998. Ciência Saúde Coletiva 2002; 7(4):743-56.

15. Longo GZ, Neves J, Castro TG, Pedroso MRO, Matos IB. Prevalência e distribuição dos fatores de risco para doenças crônicas não transmissíveis entre adultos da cidade de Lages (SC), sul do Brasil, 2007. Rev Bras Epidemiologia 2011; 14(4):698-708.

16. Malta DC, Cezário AC, Moura L, Morais Neto OL, Silva Júnior JB. A construção da vigilância e prevenção das doenças crônicas não transmissíveis no contexto do Sistema Único de Saúde. Epidemiologia Serviços de Saúde 2006; 15(1):47-65.
17. Malta DC, Bernal RIT, Almeida MCM, Ishitani LH, Girodo AM, Paixão LMMM, Oliveira MTC, Pimenta Junior FG, Silva Júnior JB. Desigualdades intraurbanas na distribuição dos fatores de risco para doenças crônicas não transmissíveis, Belo Horizonte. Rev Bras Epidemiol 2014; (3):629-41.

18. Costa LC, Thuler LCS. Fatores associados ao risco para doenças não transmissíveis em adultos brasileiros: estudo transversal de base populacional. R Bras Est Pop 2012; 29(1):133-45.

19. Monteiro CA, Cavalcante TM, Moura EC, Claro RM, Szwarcwaldc CL. Population-based evidence of a strong decline in the prevalence of smokers in Brazil (19892003). Bulletin of the World Health Organization 2007; 85(7):501-68.

20. Moura EC, Malta DC. Consumo de bebidas alcoólicas na população adulta Brasileira: características sociodemográficas e tendência. Rev Bras Epidemiol 2011; 14( Suppl1): 61-70.

21. Chor D, Faerstein E, Alves MGM, Lopes CS. How reproducible is self-reported information on exposure tosmoking, drinking, and dietary patterns? Evidence among Brazilian adults in the Pró-Saúde study. São Paulo Med J 2003; 121(2):63-66.

22. Veras RP, Parahyba MI. O anacronismo dos modelos assistenciais para os idosos na área da saúde: desaûos para o setor privado. Cadernos de Saúde Pública 2007; 23(10): 2479-89.

23. Trindade KMC, Schmitt ACB, Casarotto RA. Queixas musculoesqueléticas em uma unidade básica de saúde: implicações para o planejamento das ações em saúde e fisioterapia. FisioterPesq 2013; 20(3):228-34.

24. IBGE.Ministério do Planejamento, Orçamento e Gestão. Instituto Brasileiro de Geograûa e Estatística - IBGE Diretoria de Pesquisas Coordenação de População e Indicadores Sociais. Indicadores sociodemográficos e de saúde no Brasil. Brasília 2009.

25. Rodrigues MAP, Fachinni LA, Piccini PX, Tomasi E, Thumé E, Silveira DS, et al. Uso de serviços básicos de saúde por idosos portadores de condições crônicas, Brasil. Rev Saúde Pública 2009; 43(4):604-12.

\section{Correspondência}

Tatiana de Oliveira Sato

Departamentos de Fisioterapia e Enfermagem

Centro de Ciências Biológicas e da Saúde

Universidade Federal de São Carlos

Endereço: Rodovia Washington Luís, km 235.

São Carlos - São Paulo - Brasil - 13565-905

E-mail: tatisato@gmail.com 